November 14, $2018 \quad$ 0:7 WSPC/INSTRUCTION FILE dpas9

\title{
ENUMERATION AND DECIDABLE PROPERTIES OF AUTOMATIC SEQUENCES
}

\author{
ÉMILIE CHARLIER \\ School of Computer Science, University of Waterloo, Waterloo, ON N2L 3G1, Canada \\ emilie.charlier@ulb.ac.be \\ NARAD RAMPERSAD \\ Department of Math/Stats, University of Winnipeg, Winnipeg, MB, R3B 2E9, Canada \\ narad.rampersad@gmail.com \\ JEFFREY SHALLIT \\ School of Computer Science, University of Waterloo, Waterloo, ON N2L 3G1, Canada \\ shallit@cs.uwaterloo.ca
}

\begin{abstract}
We show that various aspects of $k$-automatic sequences - such as having an unbordered factor of length $n-$ are both decidable and effectively enumerable. As a consequence it follows that many related sequences are either $k$-automatic or $k$-regular. These include many sequences previously studied in the literature, such as the recurrence function, the appearance function, and the repetitivity index. We also give some new characterizations of the class of $k$-regular sequences. Many results extend to other sequences defined in terms of Pisot numeration systems.
\end{abstract}

\section{Introduction}

Let $\mathbf{x}=(a(n))_{n \geq 0}$ be an infinite sequence over a finite alphabet $\Delta$. We write $\mathbf{x}[i]=a(i)$, and we let $\mathbf{x}[i . . i+n-1]$ denote the factor of length $n$ beginning at position $i$.

An infinite sequence $\mathbf{x}$ is said to be $k$-automatic if it is computable by a finite automaton taking as input the base- $k$ representation of $n$, and having $a(n)$ as the output associated with the last state encountered [5].

For example, in Figure 1 we see an automaton generating the Thue-Morse sequence $\mathbf{t}=t_{0} t_{1} t_{2} \cdots=011010011001 \cdots$. The input is $n$, expressed in base 2 , and the output is the number contained in the state last reached. 


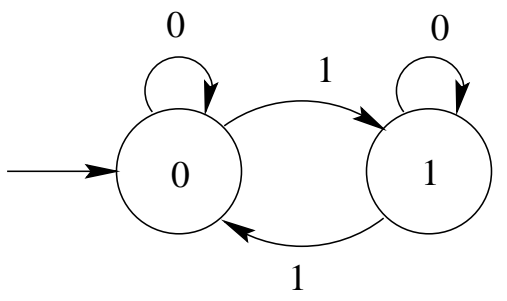

Fig. 1. A finite automaton generating a sequence

Honkala 23. showed that, given an automaton, it is decidable if the sequence it generates is ultimately periodic. Later, Leroux [26] gave a polynomial-time algorithm for the problem.

Recently, Allouche, Rampersad, and Shallit 2] found a different proof of Honkala's result using a more general technique. They showed that their technique suffices to show that the following properties (and many more) are decidable for $k$-automatic sequences $\mathbf{x}$ :

(a) Given a rational number $r>1$, whether $\mathbf{x}$ is $r$-power-free;

(b) Given a rational number $r>1$, whether $\mathbf{x}$ contains infinitely many occurrences of $r$-powers;

(c) Given a rational number $r>1$, whether $\mathbf{x}$ contains infinitely many distinct $r$-powers;

(d) Given a length $l$, whether $\mathbf{x}$ avoids palindromes of length $\geq l$.

Related results have recently been given by Halava, Harju, Kärki, and Rigo [22].

In this paper we show that many additional properties of automatic sequences are decidable using the same general technique. More significantly, we also show that related enumeration questions on automatic sequences (such as counting the number of distinct factors of length $n$ ) can be solved using a similar technique, in an entirely effective manner. As a consequence, we recover or improve results due to Mossé 27; Allouche, Baake, Cassaigne, and Damanik [1]; Currie and Saari [16]; Garel [21; Fagnot [17; and Brown, Rampersad, Shallit, and Vasiga 8].

Our main results about decidability are given in Section 2, and our main results about enumeration are given in Section 9 .

Throughout this paper, $k$ denotes a fixed integer $\geq 2$, the symbol $\mathbb{N}$ denotes the non-negative integers $\{0,1,2, \ldots\}$, and the symbol $\mathbb{N}_{\infty}$ denotes the "extended" non-negative integers $\mathbb{N} \cup\{\infty\}$.

\section{Connections with logic and new decidability results}

After the publication of [2, the third author noticed that the technique used there was, at its core, very similar to previous techniques developed by Büchi, Bruyère, 
Michaux, Villemaire, and others, involving formal logic; see, e.g., 10. This was later independently observed by the first author, as well as by Véronique Bruyère. As it turns out, the properties (a)-(d) above are decidable because they are expressible as predicates in the first-order structure $\left\langle\mathbb{N},+, V_{k}\right\rangle$, where $V_{k}(n)$ is the largest power of $k$ dividing $n$.

We briefly recall the technique discussed in [2] in the context of a particular example. Suppose we want to decide if an automatic sequence $\mathbf{x}$ is squarefree (contains no nonempty square factor). Given an automaton $M$ generating a $k$-automatic sequence $\mathbf{x}$, we create, via a series of transformations, a new automaton $M^{\prime}$ that accepts the base- $k$ representations of integers corresponding to the squares in $\mathbf{x}$. For example, $M^{\prime}$ could accept those integers corresponding to the starting position of each square, or those integers corresponding to the lengths of the squares. The operations we can use in constructing $M^{\prime}$ include digit-by-digit addition or subtraction (with carry, if necessary), comparison, and lookup of the corresponding term in $\mathbf{x}$ (which comes from simulation of $M$ ). Nondeterminism can be used to implement " $\exists$ ", and " $\forall$ " can be implemented by nondeterminism combined with suitable negations.

Ultimately, then, deciding if $\mathbf{x}$ is squarefree corresponds to verifying that $L\left(M^{\prime}\right)=\emptyset$ for the $M^{\prime}$ we construct. Deciding whether $\mathbf{x}$ contains only finitely many square occurrences corresponds to verifying that $L\left(M^{\prime}\right)$ is finite. Both can easily be done by the standard methods for automata, using depth-first or breadth-first search on the underlying state diagram of the automaton.

In this paper, we always assume that numbers are encoded in base $k$ using the digits in $\Sigma_{k}=\{0,1, \ldots, k-1\}$, The canonical encoding of $n$ is the one with no leading zeroes and is denoted $(n)_{k}$. Similarly, if $w=a_{1} \cdots a_{n} \in \Sigma_{k}^{*}$, then by $[w]_{k}$ we mean $\sum_{1 \leq i \leq n} a_{i} k^{n-i}$, the integer that $w$ represents. Often we will deal with reversed representations, where the least significant digit appears first. For example, in the reversed representation, 13 is represented in base 2 by the word 1011 .

Sometimes we will need to encode pairs, triples, or $r$-tuples of integers. We handle these by first padding the reversed representation of the smaller integer with trailing zeroes, and then coding the $r$-tuple as a word over $\Sigma_{k}^{r}$. For example, the pair $(20,13)$ could be represented in base- 2 as

$$
[0,1][0,0][1,1][0,1][1,0],
$$

where the first components spell out 00101 and the second components spell out 10110. Of course, there are other possible representations, such as

$$
[0,1][0,0][1,1][0,1][1,0][0,0],
$$

which correspond to non-canonical representations having trailing zeroes. In general, we permit these.

Thus, the main idea of [2] can be restated as follows:

Theorem 1. If we can express a property of a $k$-automatic sequence $\mathbf{x}$ using quantifiers, logical operations, integer variables, the operations of addition, subtraction, 
4 Émilie Charlier, Narad Rampersad, and Jeffrey Shallit

indexing into $\mathbf{x}$, and comparison of integers or elements of $\mathbf{x}$, then this property is decidable.

We illustrate the idea with the following new result. A word $w$ is bordered if it begins and ends with the same word $x$ with $0<|x| \leq|w| / 2$. (An example in English is ingoing, which begins and ends with ing.) Otherwise it is unbordered.

Theorem 2. Let $\mathbf{x}=a(0) a(1) a(2) \cdots$ be a $k$-automatic sequence. Then the associated infinite sequence $\mathbf{b}=b(0) b(1) b(2) \cdots$ defined by

$$
b(n)= \begin{cases}1, & \text { if } \mathbf{x} \text { has an unbordered factor of length } n \\ 0, & \text { otherwise }\end{cases}
$$

is $k$-automatic.

Proof. The sequence $\mathbf{x}$ has an unbordered factor of length $n$

iff

$\exists j \geq 0$ such that the factor of length $n$ beginning at position $j$ of $\mathbf{x}$ is unbordered

iff

there exists an integer $j \geq 0$ such that for all possible lengths $l$ with $1 \leq l \leq n / 2$, there is an integer $i$ with $0 \leq i<l$ such that the $i$ 'th letter in the supposed border of length $l$ beginning and ending the factor of length $n$ beginning at position $j$ of $\mathbf{x}$ actually differs in the $i$ 'th position

iff

there exists an integer $j \geq 0$ such that for all integers $l$ with $1 \leq l \leq n / 2$ there exists an integer $i$ with $0 \leq i<l$ such that $a(j+i) \neq a(j+n-l+i)$.

To carry out this test, we first create an NFA that given the encoding of $(j, l, n)$ guesses the base- $k$ representation of $i$, digit-by-digit, checks that $i<l$, computes $j+i$ and $j+n-l+i$ on the fly, and checks that $a(j+i) \neq a(j+n-l+i)$. If such an $i$ is found, it accepts. We then convert this to a DFA, and interchange accepting and nonaccepting states. This DFA $M_{1}$ accepts $(j, l, n)$ such that there is no $i, 0 \leq i<l$ such that $a(j+i)=a(j+n-l+i)$. We then use $M_{1}$ as a subroutine to build an NFA $M_{2}$ that on input $(j, n)$ guesses $l$, checks that $1 \leq l \leq n / 2$, and calls $M_{1}$ on the result. We convert this to a DFA and interchange accepting and nonaccepting states to get $M_{3}$. Finally, this $M_{3}$ is used as a subroutine to build an NFA $M_{4}$ that on input $n$ guesses $j$ and calls $M_{3}$.

The set of such integers $n$ then forms a $k$-automatic sequence.

Example 3. Consider the problem of determining for which lengths the ThueMorse sequence has an unbordered factor. Currie and Saari [16] proved that if $n \not \equiv 1(\bmod 6)$, then there is an unbordered factor of length $n$. (Also see [30], Lemma 4.10 and Problem 4.1.) However, this is not a necessary condition, as

$$
\mathbf{t}[39 . .69]=0011010010110100110010110100101
$$


which is an unbordered factor of length 31 . They left it as an open problem to give a complete characterization of the lengths for which $\mathbf{t}$ has an unbordered factor. Our method shows the characteristic sequence of such lengths is 2-automatic.

Further, we conjecture that there is an unbordered factor of length $n$ in $\mathbf{t}$ if and only if the base- 2 expansion of $n$ (starting with the most significant digit) is not of the form $1\left(01^{*} 0\right)^{*} 10^{*} 1$.

In principle this could be verified, purely mechanically, by our method, but we have not yet done so.

We now turn to deciding if a given automatic sequence $\mathbf{x}$ has infinite critical exponent (e.g., 24]). If a word $w$ can be written in the form $x^{n} x^{\prime}$, where $n \geq 1$ is an integer and $x^{\prime}$ is a prefix of $x$, then we say it is a fractional power with exponent $|w| /|x|$. For example, ingoing has exponent $7 / 4$. The largest such exponent is called the exponent of the word. The critical exponent of $\mathbf{x}$ is the supremum, over all finite factors $f$ of $\mathbf{x}$, of the exponent of $f$.

Theorem 4. The following question is decidable: given a k-automatic sequence, does it contain powers of arbitrarily large exponent?

Proof. $\mathbf{x}$ has powers of arbitrarily high exponent

iff

the set of pairs

$S:=\{(n, j): \exists i \geq 0$ such that for all $t$ with $0 \leq t<n$ we have $\mathbf{x}[i+t]=\mathbf{x}[i+j+t]\}$

contains pairs $(n, j)$ with $n / j$ arbitrarily large

iff

for all $i \geq 0 S$ contains a pair $(n, j)$ with $n>j \cdot 2^{i}$

iff

$L$, the set of base- $k$ encodings of pairs in $S$, contains, for each $i$, words ending in

$$
\overbrace{[*, 0][*, 0] \cdots[*, 0]}^{i}[b, 0]
$$

for some $b \neq 0$, where $*$ means any digit.

But we can easily decide if a regular language contains words ending in arbitrarily long words of this form.

In a similar fashion we can show

Theorem 5. The following question is decidable: given a $k$-automatic sequence $\mathbf{x}$, does $\mathbf{x}$ contain arbitrarily large unbordered factors?

Now we turn to questions of recurrence.

An infinite word $\mathbf{a}=(a(n))_{n \geq 0}$ is said to be recurrent if every factor that occurs at least once in a occurs infinitely often. Equivalently, a word is recurrent if and 
only if for each occurrence of a factor of $\mathbf{a}$, there exists a later occurrence of that factor in a. Equivalently, for every $n \geq 0, r \geq 1$, there exists $m>n$ such that $a(n+j)=a(m+j)$ for $0 \leq j<r$.

Similarly, an infinite word $\mathbf{a}=(a(n))_{n \geq 0}$ is said to be uniformly recurrent if every factor that occurs at least once in a occurs infinitely often, with bounded gaps between consecutive occurrences. Equivalently, a word $\mathbf{a}=(a(n))_{n \geq 0}$ is uniformly recurrent iff for every $r \geq 1$ there exists $t>0$ such that for every $n \geq 0$ there exists $m \geq 0$ with $n<m<n+t$ such that $a(n+i)=a(m+i)$ for $0 \leq i<r$.

Thus we recover the following recent result of Nicolas and Pritykin [28]:

Theorem 6. It is decidable if a k-automatic sequence is recurrent or uniformly recurrent.

We now turn to questions of factors shared by two $k$-automatic sequences. Fagnot 17. showed that it is decidable whether two such sequences $\mathbf{x}=a(0) a(1) \cdots$ and $\mathbf{y}=b(0) b(1) \cdots$ have exactly the same set of factors. This is also decidable by our methods, as follows:

The sequences $\mathbf{x}=a(0) a(1) \cdots$ and and $\mathbf{y}=b(0) b(1) \cdots$ have the same set of factors

iff

for all $i \geq 0, n \geq 1$ there exists $j \geq 0$ such that $\mathbf{x}[i . . i+n-1]=\mathbf{y}[j . . j+n-1]$

iff

for all $i \geq 0, n \geq 1$ there exists $j \geq 0$ such that for all $t, 0 \leq t<n$ we have $a(i+t)=b(j+t)$.

In a similar fashion, the question of whether the set of factors of one $k$-automatic word form a subset of the set of factors of another $k$-automatic word is decidable.

\section{Enumeration}

We now turn to questions of enumeration. A typical example of the kind of question we are interested in is, given an automatic sequence $(a(n))_{n \geq 0}$, how many distinct factors are there of length $n$ ? Our goal in the remainder of this paper is to show that these kinds of questions often have a useful answer in terms of $k$-regular sequences. A sequence $(a(n))_{n \geq 0}$ is $k$-regular if the module generated by its $k$-kernel, which is the set of all subsequences of the form

$$
\left\{\left(a\left(k^{e} n+c\right)\right)_{n \geq 0}: e \geq 0,0 \leq c<k^{e}\right\},
$$

is finitely generated \begin{tabular}{l|l|l|l|l|l}
3 & 4 & 5 & 7
\end{tabular} . The $k$-regular sequences play the same role for integer-valued sequences as the $k$-automatic sequences play for sequences over a finite alphabet. Classical examples of $k$-regular sequences include polynomials in $n$, and $s_{k}(n)$, the sum of the base- $k$ digits of $n$.

Not only does this interpretation give an explicit and efficient algorithm for computing the values of the sequence in question, it also gives a way to compute 
many related quantities that, up to now, have received extended treatments in the literature using a wide variety of techniques. Our work therefore extends and unifies many results in the literature.

In order to make our results really precise, we need several sections of preliminary definitions and results. This is what follows in Sections 4, We resume the exposition of our results in Section 9

\section{4. $k$-regular sequences}

Cobham 14 showed that a sequence $(s(n))_{n>0}$ is $k$-automatic iff its $k$-kernel is finite. Generalizing this notion, Allouche and Shallit $3 \mid 4$ introduced the notion of $k$-regular sequence over a ring $R$. A sequence is $k$-regular if the module generated by its $k$-kernel is finitely generated. In particular, Allouche and Shallit were interested in the cases of where the underlying ring is $\mathbb{Z}$ or $\mathbb{Q}$. However, as noted in the recent book of Berstel and Reutenauer [7, it makes more sense to define the $k$-regular sequences over a semiring instead of a ring. The advantage is greater generality, but at the cost of giving up part of the characterization in terms of the $k$-kernel.

Example 7. To illustrate this, consider the sequence $s_{2}(n)$ defined to be the sum of the bits in the base- 2 representation of $n$. For example, $s_{2}(27)=4$. Then $s_{2}(n)$ is 2-regular over $\mathbb{Z}$, as its 2-kernel $K$ generates a module $M$ that is generated by the sequence $s_{2}(n)$ itself and the constant sequence 1 . Indeed, we have

$$
\begin{aligned}
K & =\left\{\left(s_{2}\left(2^{e} n+a\right)\right)_{n \geq 0}: \quad e \geq 0,0 \leq a<2^{e}\right\} \\
& =\left\{\left(s_{2}(n)+s_{2}(a)\right)_{n \geq 0}: a \geq 0\right\} \\
& =\left\{\left(s_{2}(n)+c\right)_{n \geq 0}: \quad c \geq 0\right\},
\end{aligned}
$$

so that every sequence in the 2-kernel is a $\mathbb{Z}$-linear combination of $\left(s_{2}(n)\right)_{n \geq 0}$ and the constant sequence 1 . Indeed, it is even true that every sequence in $K$ is an $\mathbb{N}$-linear combination of $\left(s_{2}(n)\right)_{n \geq 0}$ and the constant sequence 1 .

In [3], the authors show that every sequence in the $k$-kernel $K$ of a $k$-regular sequence over $\mathbb{Z}$ is generated by some finite subset of $K$. For example, for $\left(s_{2}(n)\right)_{n \geq 0}$, the 2 -kernel $K$ is generated by $\left(s_{2}(n)\right)_{n \geq 0}$ and $\left(s_{2}(2 n+1)\right)_{n \geq 0}$. However, in this example, there is no finite subset $K^{\prime} \subseteq K$ such that every sequence in $K$ can be written as an $\mathbb{N}$-linear combination of the sequences in $S$. For every sequence in $K$ is of the form $s_{2}(n)+c$ with $c \geq 0$. If we take some finite subset $K^{\prime} \subseteq K$, then the sequences in $K^{\prime}$ of the form $s_{2}(n)+c$ all satisfy $c<C$ for some finite $C$. We then cannot get $s_{2}(n)+C+1$ as an $\mathbb{N}$-linear combination of the sequences in $K^{\prime}$ (as any such combination would have at least two copies of $\left.s_{2}(n)\right)$.

This means that to define ( $\mathbb{N}, k)$-regular sequences, we have to give up one characterization in terms of the kernel, given in 3 . 
8 Émilie Charlier, Narad Rampersad, and Jeffrey Shallit

\section{5. $(R, k)$-regular sequences}

In this section we give a rigorous definition of $(R, k)$-regular sequences and show that there are a number of alternative characterizations that are equivalent.

First, we give some definitions.

Let $\Sigma_{k}$ denote the alphabet $\{0,1, \ldots, k-1\}$. Let $C_{k}=\{\epsilon\} \cup\left(\Sigma_{k}-\{0\}\right) \Sigma_{k}^{*}$ denote the set of canonical base- $k$ expansions, that is, those with no leading zero. Let $R$ be a semiring. A formal series is a map $h: \Sigma^{*} \rightarrow R$. For historical reasons, $h(w)$ is often written as $(h, w)$ and $h$ itself is expressed as the formal sum $\sum_{w \in \Sigma^{*}}(h, w) w$. A formal series $h$ taking values in a semiring $R$ is said to be $R$-recognizable if $(h, w)=u \mu(w) v$ for all $w \in \Sigma^{*}$, where $\mu$ is a morphism from $\Sigma^{*}$ to the set of $n \times n$ matrices, $u$ is a $1 \times n$ matrix (or row vector), and $v$ is an $n \times 1$ matrix (or column vector), all with entries in $R$. The triple $(u, \mu, v)$ is called a linear representation of $h$.

The reader is directed to [25/32] and especially [7] for more information about recognizable series.

We recall the following standard result about recognizable series ([7, Ex. 2.1.3, p. 42):

Lemma 8. Let $R$ be a semiring, and let $f: \Sigma_{k}^{*} \rightarrow R$ be an $R$-recognizable series. Then the series $g: \Sigma_{k}^{*} \rightarrow R$ defined by $(g, w)=\left(f, w^{R}\right)$ is also $R$-recognizable.

Next, we prove a somewhat technical lemma that essentially says that we can disregard leading 0's in the representation of a word.

Lemma 9. Let $R$ be a semiring, and let $f: \Sigma_{k}^{*} \rightarrow R$ be an $R$-recognizable series. Then there exists another $R$-recognizable series $g$ such that $\left(g, 0^{i} w\right)=(f, w)$ for all $i \geq 0$ and all $w \in C_{k}$. Furthermore, there exists a linear representation $\left(u^{\prime}, \mu^{\prime}, v^{\prime}\right)$ for $g$ satisfying $u^{\prime} \mu^{\prime}(0)=u^{\prime}$.

Proof. Suppose $(u, \mu, v)$ is a rank- $n$ linear representation of $f$. Let $I_{n}$ denote the $n \times n$ identity matrix. Define $u^{\prime}, \mu^{\prime}, v^{\prime}$ as follows:

$$
\begin{aligned}
& u^{\prime}=[\overbrace{00 \cdots 0}^{n} u] \\
& \mu^{\prime}(a)= \begin{cases}{\left[\begin{array}{cc}
\mu(0) & \mathbf{0} \\
\mathbf{0} & I_{n}
\end{array}\right],} & \text { if } a=0 ; \\
{\left[\begin{array}{cc}
\mu(a) & \mathbf{0} \\
\mu(a) & \mathbf{0}
\end{array}\right],} & \text { if } a \neq 0 ;\end{cases} \\
& v^{\prime}=\left[\begin{array}{ll}
v & v
\end{array}\right]^{T},
\end{aligned}
$$

and set $g=\left(u^{\prime}, \mu^{\prime}, v^{\prime}\right)$. 
To see that this works, we will first prove the following two facts:

$$
\mu^{\prime}\left(0^{i}\right)=\left[\begin{array}{cc}
\mu\left(0^{i}\right) & \mathbf{0} \\
\mathbf{0} & I_{n}
\end{array}\right]
$$

for $i \geq 0$ and

$$
\mu^{\prime}\left(0^{i} w\right)=\left[\begin{array}{rr}
\mu\left(0^{i} w\right) & \mathbf{0} \\
\mu(w) & \mathbf{0}
\end{array}\right]
$$

for $i \geq 0$ and $w \in\left(\Sigma_{k}-\{0\}\right) \Sigma_{k}^{*}$. The claim (11) is a trivial induction, and is omitted. Let's prove (2) by induction on $|w|$. The base case is $|w|=1$. In that case $w=a$, where $a \in \Sigma_{k}-\{0\}$. From the definition we have

$$
\mu^{\prime}(w)=\mu^{\prime}(a)=\left[\begin{array}{ll}
\mu(a) & \mathbf{0} \\
\mu(a) & \mathbf{0}
\end{array}\right]
$$

so, using (1), we get

$$
\mu^{\prime}\left(0^{i} w\right)=\mu^{\prime}\left(0^{i}\right) \mu^{\prime}(w)=\left[\begin{array}{cc}
\mu\left(0^{i}\right) \mu(a) & 0 \\
\mu(a) & \mathbf{0}
\end{array}\right]=\left[\begin{array}{cc}
\mu\left(0^{i} a\right) & 0 \\
\mu(a) & \mathbf{0}
\end{array}\right],
$$

as desired. For the induction step, assume the result (2) holds for all $w^{\prime}$ with $0<$ $\left|w^{\prime}\right|<|w|$; we prove it for $w$. Write $w=a x$ with $a \in \Sigma_{k}-\{0\}$. There are two cases: (i) $x=0^{j}$ for some $j \geq 1$, and (ii) $x=0^{j} y$, where $j \geq 0$ and $y \in C_{k}$. In case (i) we have, by induction, that

$$
\mu^{\prime}(x)=\left[\begin{array}{cc}
\mu\left(0^{j}\right) & \mathbf{0} \\
\mathbf{0} & I_{n}
\end{array}\right]
$$

and hence

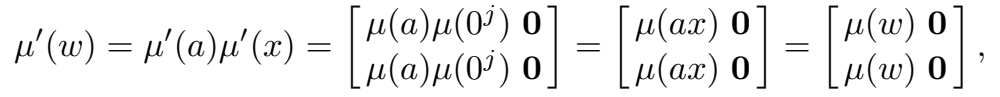

as desired. In case (ii) we have, by induction, that

$$
\mu^{\prime}(x)=\mu^{\prime}\left(0^{j} y\right)=\left[\begin{array}{rr}
\mu\left(0^{j} y\right) & 0 \\
\mu(y) & \mathbf{0}
\end{array}\right]
$$

and again we have

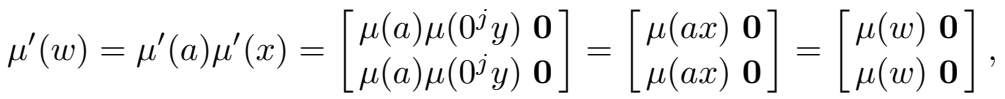

as desired.

Therefore

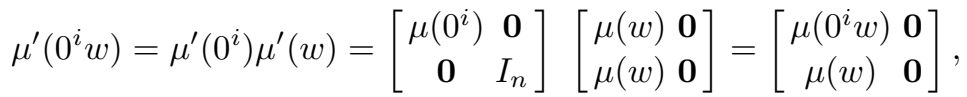

which completes the induction. 
Now that we know that (11) and (2) hold, we have, if $w=0^{i}$ for some $i \geq 0$, that

$$
\begin{aligned}
& (g, w)=u^{\prime} \mu^{\prime}(w) v^{\prime}
\end{aligned}
$$

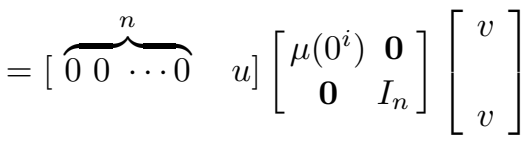

$$
\begin{aligned}
& =u v \\
& =(f, \epsilon) \text {, }
\end{aligned}
$$

as desired.

If $w=0^{i} z$ with $i \geq 0$ and $z \in\left(\Sigma_{k}-\{0\}\right) \Sigma_{k}^{*}$, then

$$
\begin{aligned}
(g, w) & =u^{\prime} \mu^{\prime}(w) v^{\prime} \\
& =[\overbrace{00 \cdots 0}^{n} u\left[\begin{array}{rr}
\mu\left(0^{i} z\right) & \mathbf{0} \\
\mu(z) & \mathbf{0}
\end{array}\right]\left[\begin{array}{l}
v \\
v
\end{array}\right] \\
& =u \mu(z) v \\
& =(f, z),
\end{aligned}
$$

as desired.

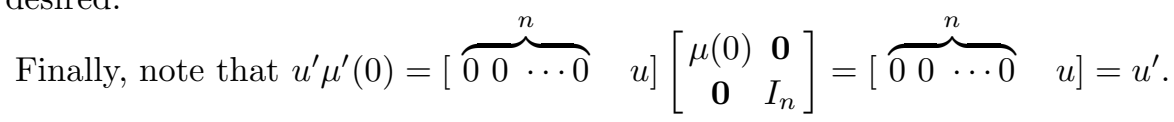

Combining the previous two lemmas, we get

Lemma 10. Let $R$ be a semiring, and let $f: \Sigma_{k}^{*} \rightarrow R$ be an $R$-recognizable series. Then there exists another $R$-recognizable series $g$ such that $\left(g, w 0^{i}\right)=(f, w)$ for all $i \geq 0$ and all $w \in C_{k}^{R}$. Furthermore, there exists a linear representation $\left(u^{\prime}, \mu^{\prime}, v^{\prime}\right)$ for $g$ satisfying $\mu^{\prime}(0) v^{\prime}=v^{\prime}$.

We are now ready to state our equivalence theorem. This result can be viewed as an expanded version of [7, Prop. 1.1, p. 84 .

Theorem 11. Let $(f(n))_{n \geq 0}$ be a sequence taking values in a semiring $R$. The following are equivalent.

(a) There exist finitely many sequences $\left(f_{1}(n)\right)_{n \geq 0}, \ldots,\left(f_{r}(n)\right)_{n \geq 0}$ such that

(i) $(f(n))_{n \geq 0}$ is an R-linear combination of the $f_{i}$; and

(ii) for each $i$ and $a$ with $1 \leq i \leq r$, and $0 \leq a<k$, the subsequence $\left(f_{i}(k n+a)\right)_{n \geq 0}$ is an R-linear combination of the $\left(f_{i}(n)\right)_{n \geq 0}$.

(b) There exist finitely many sequences $\left(f_{1}(n)\right)_{n \geq 0}, \ldots,\left(f_{r}(n)\right)_{n \geq 0}$ and $k$ matrices $B_{0}, B_{1}, \ldots, B_{k-1}$ with entries in $R$ such that if

$$
V(n)=\left(\begin{array}{c}
f_{1}(n) \\
\vdots \\
f_{r}(n)
\end{array}\right),
$$


then $V(k n+a)=B_{a} V(n)$ for $0 \leq a<k$. and there exists a vector $z \in R^{1 \times r}$ such that $f(n)=z \cdot V(n)$.

(c) There exist a matrix-valued morphism $\mu: \Sigma_{k}^{*} \rightarrow R^{r \times r}$ and vectors $u, v$ with entries in $R$, such that $\mu(0) v=v$ and $f(n)=u \mu\left(w^{R}\right) v$ for all $w \in \Sigma_{k}^{*}$ with $[w]_{k}=n$.

(d) There exist a matrix-valued morphism $\rho: \Sigma_{k}^{*} \rightarrow R^{s \times s}$ and vectors $u^{\prime}, v^{\prime}$ with entries in $R$, such that $u^{\prime}=u^{\prime} \rho(0)$ and $f(n)=u^{\prime} \rho(w) v^{\prime}$ for all $w \in \Sigma_{k}^{*}$ with $[w]_{k}=n$.

(e) There is an R-recognizable series $d$ such that $(d, w)=f\left([w]_{k}\right)$ for all $w \in$ $C_{k}$.

(f) The mapping $(h, w):=f\left([w]_{k}\right)$ defines an R-recognizable series.

(g) The mapping $\left(h^{\prime}, w\right):=f\left(\left[w^{R}\right]_{k}\right)$ defines an $R$-recognizable series.

(h) There is an $R$-recognizable series $p$ such that $(p, w)=f\left(\left[w^{R}\right]_{k}\right)$ for all $w \in C_{k}^{R}$.

Proof. (a) $\Longrightarrow$ (b): Since each $f_{i}(k n+a)$ is an $R$-linear combination of the $f_{i}$, we can express this as the matrix product $V(k n+a)=B_{a} V(n)$. Since $f(n)$ is an $R$-linear combination of the $f_{i}(n)$, we can express this as $f(n)=z \cdot V(n)$ for a suitable vector $z$.

(b) $\Longrightarrow$ (c): In fact we can take $v=V(0), \mu(a)=B_{a}$ for $0 \leq a<k$, and $u=z$. Let us prove by induction on $n$ that $V(n)=\mu\left((n)_{k}^{R}\right) V(0)$. The base case is $n=0$. Then $(n)_{k}=\epsilon$, so $\mu\left((n)_{k}^{R}\right)=I$, the identity matrix, and $V(0)=I \cdot V(0)$.

Now assume the result is true for all $n^{\prime}<n$, and we prove it for $n$. Write $n=k n^{\prime}+a$ for $0 \leq a<k$. Then by induction $V\left(n^{\prime}\right)=\mu\left(\left(n^{\prime}\right)_{k}^{R}\right) V(0)$. Then $V(n)=V\left(k n^{\prime}+a\right)=\mu(a) V\left(n^{\prime}\right)=\mu(a) \mu\left(\left(n^{\prime}\right)_{k}^{R}\right) V(0)=\mu\left((n)_{k}^{R}\right) V(0)$.

We have $f(n)=z V(n)$. Furthermore, from $V(k n+a)=\mu(a) V(n)$ with $k=$ $0, n=0, a=0$, we get $v=\mu(0) v$.

Finally, if $w \in \Sigma_{k}^{*}$ is such that $[w]_{k}=n$, then $w^{R}=(n)_{k}^{R} 0^{i}$ for some $i \geq 0$. Because $v=\mu(0)^{i} v$, we have $u \mu\left(w^{R}\right) v=u \mu\left((n)_{k}^{R}\right) \mu(0)^{i} v=u \mu\left((n)_{k}^{R}\right) v=f(n)$.

(c) $\Longrightarrow$ (d): Let $\rho(i):=\mu(i)^{T}, u^{\prime}:=v^{T}$, and $v^{\prime}:=u^{T}$. Then from (c) we get $f(n)=u^{\prime} \rho\left((n)_{k}\right) v^{\prime}$. Furthermore, from $v=\mu(0) v$ we get $v^{T}=v^{T} \mu(0)^{T}$, and so

$$
u^{\prime}=u^{\prime} \rho(0) .
$$

Let $w$ be any word such that $[w]_{k}=n$. Then we can write $w=0^{i}(n)_{k}$ for some $i \geq 0$. Then $u^{\prime}=u^{\prime} \rho(0)^{i}$ from (3), and hence $f(n)=u^{\prime} \rho(w) v^{\prime}$, as desired.

$(\mathrm{d}) \Longrightarrow(\mathrm{e})$ : We can take $d=\left(u^{\prime}, \rho, v^{\prime}\right)$.

(e) $\Longrightarrow$ (f): Let $d$ be an $R$-recognizable series such that $(d, w)=f\left([w]_{k}\right)$ for all words $w \in C_{k}$. Now apply Lemma 9; we obtain a new $R$-recognizable series $h$ with 
$\left(h, 0^{i} x\right)=(d, x)$ for all $i \geq 0$ and all $x \in C_{k}$. Let $w \in \Sigma_{k}^{*}$. Then we can write $w=0^{j} x$, where $x \in C_{k}$. Then $(h, w)=\left(h, 0^{j} x\right)=(d, x)=f\left([x]_{k}\right)=f\left(\left[0^{j} x\right]_{k}\right)=f\left([w]_{k}\right)$.

(f) $\Longrightarrow(\mathrm{g})$ : Using Lemma 8, if $h^{\prime}$ is the series defined by $\left(h^{\prime}, w\right)=\left(h, w^{R}\right)$, then $h^{\prime}$ is also $R$-recognizable. We have $\left(h^{\prime}, w\right)=\left(h, w^{R}\right)=f\left(\left[w^{R}\right]_{k}\right)$.

$(\mathrm{g}) \Longrightarrow(\mathrm{h})$ : Trivial.

(h) $\Longrightarrow$ (a): By Lemma 10 there exists an $R$-recognizable series $p^{\prime}$ with linear representation $\left(c^{\prime}, \gamma^{\prime}, d^{\prime}\right)$ such that $\left(p^{\prime}, w 0^{i}\right)=(p, w)$ for all $i \geq 0$ and all $w \in C_{k}^{R}$. Furthermore, $\gamma^{\prime}(0) d^{\prime}=d^{\prime}$.

Define the sequences $\left(f_{i}(n)\right)_{n \geq 0}$ as follows:

$$
\left[\begin{array}{c}
f_{1}(n) \\
f_{2}(n) \\
\vdots \\
f_{t}(n)
\end{array}\right]=\gamma^{\prime}\left((n)_{k}^{R}\right) \cdot d^{\prime}
$$

Then

$$
\left[\begin{array}{c}
f_{1}(k n+a) \\
f_{2}(k n+a) \\
\vdots \\
f_{t}(k n+a)
\end{array}\right]=\gamma^{\prime}\left((k n+a)_{k}^{R}\right) \cdot d^{\prime}
$$

If $(a, n) \neq(0,0)$ then

$$
\begin{aligned}
\gamma^{\prime}\left((k n+a)_{k}^{R}\right) \cdot d^{\prime} & =\gamma^{\prime}\left(a \cdot(n)_{k}^{R}\right) \cdot d^{\prime} \\
& =\gamma^{\prime}(a) \gamma^{\prime}\left((n)_{k}^{R}\right) \cdot d^{\prime} \\
& =\gamma^{\prime}(a)\left[\begin{array}{c}
f_{1}(n) \\
f_{2}(n) \\
\vdots \\
f_{t}(n)
\end{array}\right],
\end{aligned}
$$

which expresses each $f_{i}(k n+a)$ as a linear combination of $f_{1}(n), f_{2}(n), \ldots, f_{t}(n)$.

If $(a, n)=(0,0)$, then from (4) we get

$$
\left[\begin{array}{c}
f_{1}(k n+a) \\
f_{2}(k n+a) \\
\vdots \\
f_{t}(k n+a)
\end{array}\right]=\gamma^{\prime}\left((0)_{k}^{R}\right) \cdot d^{\prime}=\gamma^{\prime}(\epsilon) \cdot d^{\prime}=d^{\prime}=\gamma^{\prime}(0) \cdot d^{\prime}=\gamma^{\prime}(a)\left[\begin{array}{c}
f_{1}(n) \\
f_{2}(n) \\
\vdots \\
f_{t}(n)
\end{array}\right] \text {. }
$$

Furthermore,

$$
f(n)=\left(p,(n)_{k}^{R}\right)=c^{\prime} \cdot \gamma^{\prime}\left((n)_{k}^{R}\right) \cdot d^{\prime}=c^{\prime} \cdot\left[\begin{array}{c}
f_{1}(n) \\
f_{2}(n) \\
\vdots \\
f_{t}(n)
\end{array}\right]
$$


which expresses $f(n)$ as a linear combination of $f_{1}(n), f_{2}(n), \ldots, f_{t}(n)$.

We can now formally define $k$-regular sequences over a semiring.

Definition 12. Suppose $R$ is a semiring, and $f: \mathbb{N} \rightarrow R$ is a sequence with values in $R$. If any of the conditions (a)-(h) in Theorem 11 hold, then we say that $f$ is $(R, k)$-regular.

Corollary 13. If $f$ is a sequence such that $f(n)$ is an R-linear combination of some finite subset of its $k$-kernel, then it is $(R, k)$-regular.

Proof. Follows from Theorem 11(a).

However, unlike the case of $(\mathbb{Z}, k)$ - or $(\mathbb{Q}, k)$-regular sequences, the converse to Corollary 13 does not hold, as we have seen above in Example 7.

\section{N-recognizable series}

In this section, our semiring is $R=\mathbb{N}$, the non-negative integers. We prove a characterization of $\mathbb{N}$-recognizable series in terms of automata and transducers (Theorem 14, below).

We recall the notion of nondeterministic finite automaton (NFA): it is a 5 -tuple $M=\left(Q, \Sigma, \delta, q_{0}, F\right)$, where $Q$ is a set of states, $\Sigma$ is a finite alphabet, $q_{0}$ is the initial state, and $F \subseteq Q$ is the set of final states, and $\delta: Q \times \Sigma \rightarrow 2^{Q}$ is the transition function, extended to $Q \times \Sigma^{*}$ in the obvious way. A path labeled $w=a_{1} \cdots a_{n}$ in an NFA is a sequence of states $\left(p_{0}, p_{1}, \ldots, p_{n}\right)$ such that $p_{i+1} \in \delta\left(p_{i}, a_{i+1}\right)$ for $0 \leq i<n$. It is an accepting path if $p_{0}=q_{0}$ and $p_{n} \in F$.

We will also be concerned with nondeterministic uniform finite-state transducers. Such a transducer produces an output of the same length for every input symbol. Formally, such a transducer $T=\left(Q, \Sigma, \Delta, E, q_{0}, F\right)$, where $E \subseteq Q \times \Sigma \times \Delta^{l} \times Q$ is the set of permissible transitions. A transition $\left(q_{i}, a, y, q_{j}\right)$ means that if the transition is in state $q_{i}$ then on input $a$ it has the option (nondeterministically) of outputting $y$ and entering state $q_{j}$. The output of $T$ on input $w$ is the set of all words formed by concatenating the outputs on a path labeled $w$ from $q_{0}$ to some state of $F$.

Finally, given words $w=a_{1} a_{2} \cdots a_{n}$ and $x=b_{1} b_{2} \cdots b_{n}$ of the same length, but defined over possibly different alphabets (say, $\Sigma$ and $\Delta$, respectively), we define the word $w \times x$ to be the word $z=\left[a_{1}, b_{1}\right]\left[a_{2}, b_{2}\right] \cdots\left[a_{n}, b_{n}\right]$ over the alphabet $\Sigma \times \Delta$. In this case, we define the projection maps $\pi_{1}(z)=w$ and $\pi_{2}(z)=x$.

Theorem 14. Let $f: \Sigma^{*} \rightarrow \mathbb{N}$ be a formal series with $(f, \epsilon)=0$. Then the following are equivalent.

(a) $f$ is $\mathbb{N}$-recognizable.

(b) There exists an NFA $M=\left(Q, \Sigma, \delta, q_{0}, F\right)$ such that for all $w \in \Sigma^{*}$, there are exactly $(f, w)$ paths labeled $w$ from $q_{0}$ to a state of $F$. 
(c) There is an alphabet $\Delta$ and a regular language $L \subseteq(\Sigma \times \Delta)^{*}$ such that

$$
(f, w)=\left|\left\{z \in L: \pi_{1}(z)=w\right\}\right|
$$

for all words $w$.

(d) There is an alphabet $\Delta$ and a (nondeterministic) 1-uniform finite-state transducer $T: \Sigma^{*} \rightarrow \Delta^{*}$ such that $(f, w)=|T(w)|$ for all words $w$.

Proof. (a) $\Longrightarrow$ (b): Since $f$ is recognizable, there is a matrix representation $(u, \mu, v)$ such that $(f, w)=u \mu(w) v$ for all $w \in \Sigma^{*}$. By an exercise in [31, Ex. III.3.3, p. 426, we can, without loss of generality, assume that $u=\left[\begin{array}{llll}1 & \overbrace{0}^{0} \cdots & \cdots\end{array}\right]$ and $v=\overbrace{\begin{array}{llll}0 & \cdots & \cdots & 0\end{array}}^{n-1} 1]^{T}$ for some $n \geq 2$. For completeness, we give the proof here:

Given a rank- $t$ representation $(u, \mu, v)$, we produce a new rank- $(t+2)$ representation $\left(u^{\prime}, \mu^{\prime}, v^{\prime}\right)$ defined as follows:

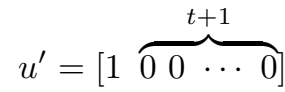

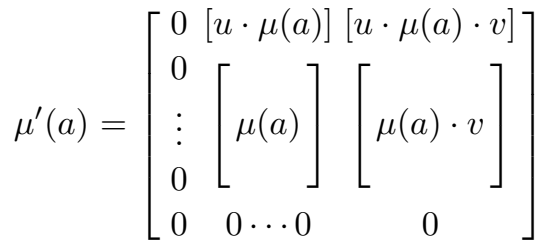

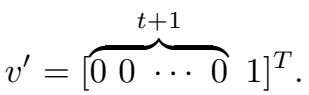

Now an easy induction on $|w|$ shows that, for $|w| \geq 1$, that

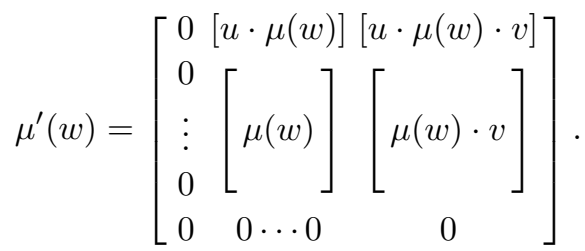

It follows that, for $w \neq \epsilon, u^{\prime} \mu^{\prime}(w) v^{\prime}=u \mu(w) v$. For $w=\epsilon$, we have $u^{\prime} \mu^{\prime}(w) v^{\prime}=0$. This completes the proof of the exercise.

Now that this somewhat technical point has been handled, we turn to the idea of the construction. The desired interpretation is that $\mu(w)_{i, j}$ should count the number of paths labeled $w$ from state $i$ to state $j$. However, this is not sensible if $a$ is a single symbol, as there is either one directed edge in the automaton from $i$ to $j$ labeled $a$, or none. To get around this problem, we make multiple copies of each state, and create a transition from $i$ to $\mu(w)_{i, j}$ copies of state $j$. 
From the rank- $n$ linear representation for $f$, namely $(u, \mu, v)$, we create an NFA $M=\left(Q, \Sigma, \delta, q_{0}, F\right)$ with $(f, w)$ paths labeled $w$. Let $m$ be the maximum entry in all the $\mu(a), a \in \Sigma$. Define

$$
\begin{aligned}
Q & =\{[i, j]: 1 \leq i \leq n, 1 \leq j \leq m\} \\
q_{0} & =[1,1] \\
F & =\{[n, s]: 1 \leq s \leq m\} \\
\delta([i, j], a) & =\left\{[r, s]: 1 \leq r \leq n, 1 \leq s \leq \mu(a)_{i, r}\right\},
\end{aligned}
$$

where by $\mu(a)_{i, r}$ we mean the entry in row $i$ and column $r$ of the matrix $\mu(a)$.

To see that this works, let $P_{i, j, r}(w)$ denote the number of paths labeled $w$ from $[i, j]$ to some member of $\{[r, s]: 1 \leq s \leq m\}$. We claim that

$$
P_{i, j, r}(w)=\mu(w)_{i, r}
$$

for all $i, j, r, w$ such that $1 \leq i, r \leq n, 1 \leq j \leq m$, and $w \in \Sigma^{*}$.

The proof is by induction on $|w|$. The base case is $|w|=0$. In this case

$$
\mu(w)_{i, r}= \begin{cases}1, & \text { if } i=r ; \\ 0, & \text { otherwise. }\end{cases}
$$

and the only path of length 0 from state $[i, j]$ is to $[i, j]$ itself, so $P_{i, j, r}(w)=\mu(w)_{i, r}$.

Now assume (6) holds for all $\left|w^{\prime}\right|<|w|$; we prove it for $w$. Write $w=a x$ with $a \in \Sigma$. Break the path labeled $w$ into two pieces, one labeled $a$ and the other labeled $x$. Then

$$
\begin{aligned}
P_{i, j, r}(a x) & =\sum_{\substack{i^{\prime}, j^{\prime} \\
\left[i^{\prime}, j^{\prime}\right] \in \delta([i, j], a)}} P_{i^{\prime}, j^{\prime}, r}(x) \\
& =\sum_{\substack{i^{\prime}, j^{\prime} \\
\left[i^{\prime}, j^{\prime}\right] \in \delta([i, j], a)}} \mu(x)_{i^{\prime}, r} \quad \text { (by induction) } \\
& =\sum_{i^{\prime}} \mu(a)_{i, i^{\prime}} \cdot \mu(x)_{i^{\prime}, r} \\
& =\mu(a x)_{i, r},
\end{aligned}
$$

which completes the induction.

Thus $u \cdot \mu(w) \cdot v=\mu(w)_{1, n}=P_{1,1, n}(w)$, as desired.

(b) $\Longrightarrow$ (c): Given the NFA $M=\left(Q, \Sigma, \delta, q_{0}, F\right)$, we take $\Delta=Q$ and define

$L=\left\{w \times x: w \in \Sigma^{*}, x \in \Delta^{*}\right.$ such that $q_{0} x$ is an accepting path for $\left.w\right\}$.

Since there are $(f, w)$ accepting paths for $w$ in $M$, and each such path begins with $q_{0}$, the result follows. Clearly $L$ is regular, as it can be accepted by a simple modification of $M$.

(c) $\Longrightarrow$ (d): Consider a DFA $M$ accepting $L$. We construct a transducer $T$ with the same set of states, initial state, and set of final states as $M$. For each transition 
in $M$ of the form $\delta\left(q_{i},[a, b]\right)=q_{j}$, we define a transition in $T$ from $q_{i}$ to $q_{j}$ with input $a$ and output $b$. It follows that on input $w$, the transducer $T$ outputs all those $x$ of the same length for which $w \times x \in L$.

(d) $\Longrightarrow$ (a): Given such a transducer $T=\left(Q, \Sigma, \Delta, E, q_{1}, F\right)$, we define the matrix representation $(u, \mu, v)$ for the series $f$ as follows: if $Q=\left\{q_{1}, \ldots, q_{n}\right\}$, then $u=$

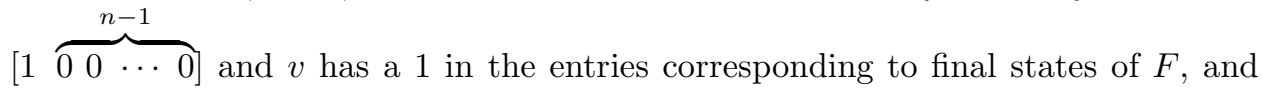
0 elsewhere. Since $|T(\epsilon)|=0$ by hypothesis, it must be that $q_{1} \notin F$. Now define $\mu(a)_{i, j}$ to be the number of symbols $b$ such that $\left(q_{i}, a, b, q_{j}\right) \in E$.

Open Problem 15. The preceding theorem would be true if we replace the 1uniform finite-state transducer with any transducer where no two different paths labeled $w$ give the same output. One way to ensure this is that the output labels form a code, and this is clearly true if the outputs are all of the same length. What happens if the output labels do not form a code? Is the result still true?

Remark 16. Carpi and Maggi 13] defined the class of $k$-synchronized sequences, a class which contains the $k$-automatic sequences and is properly contained in the class of $k$-regular sequences. A sequence $\left(u_{n}\right)_{n \geq 0}$ is $k$-synchronized if the relation $\left\{\left((n)_{k},\left(u_{n}\right)_{k}\right): n \geq 0\right\}$ is a right-synchronized rational relation. Roughly speaking, this means that the relation is realized by a length-preserving rational transduction, except that we also permit the presence of "padding" symbols at the end of one or the other component of the input. Our transducer-based characterization, combined with Theorem 11, characterizes the more general class of $k$-regular sequences.

In the usual case where $|\Sigma| \geq 2$, we can take $\Delta$ to be $\Sigma^{l}$ for a suitable $l$, as the following theorem shows.

Theorem 17. Let $f: \Sigma^{*} \rightarrow \mathbb{N}$ be a formal series with $|\Sigma| \geq 2$ and $(f, \epsilon)=0$. Then the following are equivalent.

(a) $f$ is $\mathbb{N}$-recognizable.

(b) There is an integer $l \geq 1$ and a regular language $L \subseteq\left(\Sigma \times \Sigma^{l}\right)^{*}$ such that

$$
(f, w)=\left|\left\{z \in L: \pi_{1}(z)=w\right\}\right| .
$$

for all words $w$.

(c) There is an integer $l \geq 1$ and a (nondeterministic) l-uniform finite-state transducer $T: \Sigma^{*} \rightarrow \Sigma^{*}$ such that $(f, w)=|T(w)|$.

Proof. Just like the proof of Theorem 14. The only difference is that we need to choose $l$ large enough so that $|\Sigma|^{l} \geq|\Delta|$; then we just use elements of $\Sigma^{l}$ instead of those in $\Delta$. 


\section{7. $\mathbb{N}_{\infty}$-recognizable series}

In this section, we consider the case where the underlying semiring is $R=\mathbb{N}_{\infty}$, the extended non-negative integers. Roughly speaking, this extension corresponds to the case where a nondeterministic finite automaton or transducer is extended by allowing $\epsilon$-transitions.

In addition to the usual interpretation for addition and multiplication of natural numbers, we need the following additional rules that turn $\mathbb{N}_{\infty}$ into a semiring:

(i) $a+\infty=\infty+a=\infty$ for all $a \in \mathbb{N}_{\infty}$;

(ii) $a \cdot \infty=\infty \cdot a=\infty$ for all $a \neq 0$;

(iii) $0 \cdot \infty=\infty \cdot 0=0$.

Matrices and vectors with entries in $\mathbb{N}_{\infty}$ can now be multiplied using the usual rules for such multiplication, in addition to the rules (i)-(iii), as needed.

Let $L$ be a regular language. The characteristic series of $L$, denoted $\chi_{L}$, is the formal series such that $\left(\chi_{L}, w\right)=1$ if $w \in L$ and 0 otherwise. The Hadamard product of two series, $h \odot h^{\prime}$, is the term-by-term product, $\left(h \odot h^{\prime}\right)(w)=h(w) h^{\prime}(w)$. The essential lemma is the following:

Lemma 18. Given a recognizable formal series $f$ over $\mathbb{N}_{\infty}$, we can express it as

$$
f=\chi_{\bar{L}} \odot g+\chi_{L} \cdot \infty,
$$

where $g$ is a recognizable formal series over $\mathbb{N}$ and $L$ is a regular language. Furthermore, in the sum, we never add $\infty$ to a value other than 0.

Proof. There are two main ideas. The first is that the language $L=\{w:(f, w)=$ $\infty\}$ is regular. The second is that $g$ can be taken to be a modification of $f$ with all occurrences of $\infty$ removed.

First, the construction of $L$. This is essentially that given in Salomaa and Soittola 32, p. 40, Exercise 5.

We create a new finite semiring $R^{\prime}=\{0, p, \infty\}$ where the addition and multiplication rules are given as follows:

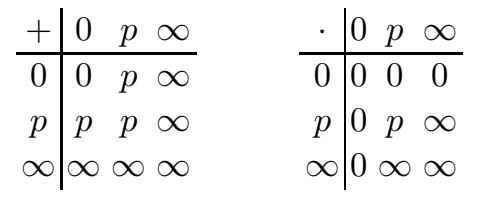

Here $0, p, \infty$ should be treated as formal symbols, but the intent is that the symbol $p$ denotes "some positive integer". Now we define a morphism of semirings $\mathbb{N}_{\infty} \rightarrow R^{\prime}$ as follows:

$$
\begin{aligned}
\tau(0) & =0 \\
\tau(i) & =p, \quad \text { for } 0<i<\infty \\
\tau(\infty) & =\infty
\end{aligned}
$$


It is now easy to check that for all $a, b \in \mathbb{N}_{\infty}$ we have $\tau(a b)=\tau(a) \cdot \tau(b)$ and $\tau(a+b)=\tau(a)+\tau(b)$, where the operations on the right-hand-side are those in $R^{\prime}$. We extend $\tau$ to apply to vectors and matrices by applying $\tau$ to each entry.

Next, we consider the formal series $f^{\prime}:=\tau \circ f$, which takes its values in $R^{\prime}$. It follows from above that $f^{\prime}(w)=\hat{u} \hat{\mu}(w) \hat{v}$, where $\hat{u}=\tau(u), \hat{v}=\tau(v)$, and $\hat{\mu}=\tau \circ \mu$.

Now we can create a deterministic finite automaton $M=\left(Q, \Sigma, \delta, q_{0}, F\right)$ that essentially computes the series $f^{\prime}$. We do this by letting $Q$ be the set of all possible $1 \times n$ row vectors over $R^{\prime}$, letting $q_{0}=\hat{u}$, and defining the transitions $\delta(q, a)=q \cdot \hat{\mu}(a)$. If we define $\varphi(t)=t \cdot \hat{v}$, then an easy induction gives that $\delta\left(q_{0}, w\right)=\hat{u} \cdot \hat{\mu}(w)$, and hence $\varphi\left(\delta\left(q_{0}, w\right)\right)=\hat{u} \cdot \hat{\mu}(w) \cdot \hat{v}=f^{\prime}(w)$, as desired.

We can now define $L$. Let $F$, the set of final states of $M$, be given by

$$
F=\{t \in Q: t \cdot \hat{v}=\infty\}
$$

Then $L=L(M)$. By construction, we have the following equivalences: $w \in L \Leftrightarrow$ $\left(f^{\prime}, w\right)=\infty \Leftrightarrow(f, w)=\infty$.

Now we turn to the construction of $g$. Let $(u, \mu, v)$ be a linear representation for $f$. Define a map $\xi: \mathbb{N}_{\infty} \rightarrow \mathbb{N}$ as follows:

$$
\xi(i)= \begin{cases}i, & \text { if } i \in \mathbb{N} \\ 0, & \text { if } i=\infty\end{cases}
$$

and extend $\xi$ to apply element-by-element to vectors and matrices in the obvious way. Let $g=\left(u^{\prime}, \mu^{\prime}, v^{\prime}\right)$, where $u^{\prime}=\xi(u), \mu^{\prime}(a)=\xi(\mu(a))$ for each $a \in \Sigma$, and $v^{\prime}=\xi(v)$. The series $g$ is created by replacing each occurrence of $\infty$ in $u, \mu$, and $v$ with 0 . Then $g$ is evidently $\mathbb{N}$-recognizable, and we claim that $(f, w) \neq \infty \Longrightarrow$ $(f, w)=(g, w)$. To see this, note that if $(f, w) \neq \infty$, then in the calculation $u \cdot \mu(w) \cdot v$ any occurrences of $\infty$ that arise must eventually be multiplied by 0 , yielding 0 . Then replacing $\infty$ with 0 has no effect, since any multiplication involving 0 will also yield 0 . (Note that we are not claiming anything about those $w$ for which $(f, w)=\infty$; the corresponding values of $g$ could be anything.)

It now follows that $f=\chi_{\bar{L}} \odot g+\chi_{L} \cdot \infty$.

We get the following two corollaries.

Corollary 19. If $f: \Sigma^{*} \rightarrow \mathbb{N}$ is an $\mathbb{N}_{\infty}$-recognizable series, then it is $\mathbb{N}$ recognizable.

Proof. From Lemma 18, we have $f=\chi_{\bar{L}} \odot g+\chi_{L} \cdot \infty$, where $g$ is an $\mathbb{N}$-recognizable formal series and $L$ is a regular language. Since $f\left(\Sigma^{*}\right) \subseteq \mathbb{N}$ and from the proof of Lemma 18, we may choose $L=\emptyset$. So $f=g$.

Corollary 20. Given a recognizable formal series $f$ over $\mathbb{N}_{\infty}$, with linear representation $(u, \mu, v)$, there exists another linear representation $(p, \beta, q)$ such that the only entries equal to $\infty$ lie in $p$. 
Proof. First, by a well-known result on the Hadamard product (e.g., 33] and [7, p. 15 , the formal series $g^{\prime}:=\chi_{\bar{L}} \odot g$ constructed in Lemma 18 is recognizable (over $\mathbb{N}$ ). So $g^{\prime}$ has a linear representation $(u, \mu, v)$ that contains no entries of $\infty$. Similarly, since $L$ is a regular language, the characteristic series $\chi_{L}$ has a linear representation $(r, \alpha, s)$, where the entries of $r, \alpha$, and $s$ are all either 0 or 1 . From this we can form a new linear representation $(p, \beta, q)$ for $f$, via a direct sum construction, as follows:

$$
\begin{aligned}
p & =\left[\begin{array}{ll}
u & r \cdot \infty
\end{array}\right] \\
\beta(a) & =\left[\begin{array}{cc}
\mu(a) & \mathbf{0} \\
\mathbf{0} & \alpha(a)
\end{array}\right] \\
q & =\left[\begin{array}{ll}
v & s
\end{array}\right]^{T} .
\end{aligned}
$$

Here, $\mathbf{0}$ represents a matrix of 0 's of the appropriate size.

A routine induction shows that $\beta(w)$ contains $\mu(w)$ in the upper left and $\alpha(w)$ in the lower right, from which the result follows.

\section{Characterizations of $\mathbb{N}_{\infty}$-recognizable series}

Just as the $\mathbb{N}$-recognizable series have a number of different interpretations in terms of automata and transducers, as we saw in Section [6 so do the $\mathbb{N}_{\infty}$-recognizable series; the difference is that we need to allow $\epsilon$-transitions.

Theorem 21. Let $f: \Sigma^{*} \rightarrow \mathbb{N}_{\infty}$ be a formal series. Then the following are equivalent:

(a) $f$ is $\mathbb{N}_{\infty}$-recognizable;

(b) There exists an NFA- $\epsilon M=\left(Q, \Sigma, \delta, q_{0}, F\right)$ such that, for all $w \in \Sigma^{*}$, there are exactly $(f, w)$ paths labeled $w$ from $q_{0}$ to a state of $F$;

(c) There is an alphabet $\Delta$, a symbol $\mathrm{B} \notin \Sigma$ and a regular language $L \subseteq((\Sigma \cup$ $\{\mathrm{B}\}) \times \Delta)^{*}$ such that

$$
(f, w)=\left|\left\{z \in L: \tau\left(\pi_{1}(z)\right)=w\right\}\right|,
$$

where $\tau$ is the morphism that maps a to a for $a \in \Sigma$ and $\mathrm{B}$ to $\epsilon$;

(d) There is an alphabet $\Delta$, a symbol $\mathrm{B} \notin \Sigma$, and a regular language $L \subseteq$ $((\Sigma \cup\{\mathrm{B}\}) \times \Delta)^{*}$ such that

$$
(f, w)=\left|\left\{z \in L: \pi_{1}(z) \in w \mathrm{~B}^{*}\right\}\right| .
$$

(e) There is an alphabet $\Delta$ and a nondeterministic finite-state transducer $T$, with inputs of a single letter or $\epsilon$ on every transition, and outputs of a single letter on every transition, such that $(f, w)=|T(w)|$.

Proof. We prove the implications in the order (a) $\Longrightarrow(\mathrm{d}) \Longrightarrow(\mathrm{e}) \Longrightarrow$ (c) $\Longrightarrow(\mathrm{b}) \Longrightarrow(\mathrm{a})$. 
(a) $\Longrightarrow$ (d): By Theorem 18, we know that $f=\chi_{\overline{L_{1}}} \odot g+\chi_{L_{1}} \cdot \infty$, where $L_{1} \subseteq \Sigma^{*}$ is a regular language and $g$ is an $\mathbb{N}$-recognizable series. Define $g^{\prime}:=\chi_{\overline{L_{1}}-\{\epsilon\}} \odot g$; then $g^{\prime}$ is an $\mathbb{N}$-recognizable series with $\left(g^{\prime}, \epsilon\right)=0$, so we can apply the implication (a) $\Longrightarrow$ (c) in Theorem 14 to $g^{\prime}$ to get an alphabet $\Delta$ and a regular language $L_{2} \subseteq(\Sigma \times \Delta)^{*}$ such that

$$
\left(g^{\prime}, w\right)=\left|\left\{z \in L_{2}: \pi_{1}(z)=w\right\}\right|
$$

for all words $w \neq \epsilon$. Let $a$ be an arbitrarily chosen, fixed symbol of $\Delta$, and consider the language $L_{3}$ defined by

$L_{3}=\left(\bigcup_{0 \leq i<(f, \epsilon)}[\mathrm{B}, a]^{i}\right) \cup\left\{z \in((\Sigma \cup\{\mathrm{B}\}) \times \Delta)^{*}: \pi_{1}(z) \in\left(L_{1}-\{\epsilon\}\right) \cdot \mathrm{B}^{*}\right.$ and $\left.\pi_{2}(z) \in a^{*}\right\}$.

It is easy to see that $L_{3}$ is regular, as each term of the big union is regular. For the second term, we can, given a DFA for $L_{1}-\{\epsilon\}$, modify it by

- changing each transition on any letter $b$ to a transition on $[b, a]$

- adding transitions out of each accepting state on $[\mathrm{B}, a]$ to a new final state $q$ and

- adding a self-loop labeled $[\mathrm{B}, a]$ from $q$ to itself.

Let $L:=L_{2} \cup L_{3}$. Then $L$ is regular and, by construction, $(f, w)=\mid\{z \in L$ : $\left.\pi_{1}(z) \in w \mathrm{~B}^{*}\right\} \mid$.

(d) $\Longrightarrow$ (e): Given a DFA $M$ for $L$, say $M=\left(Q, \Sigma^{\prime}, \delta, q_{0}, F\right)$ where $\Sigma^{\prime}=$ $(\Sigma \cup\{\mathrm{B}\}) \times \Delta$, we create the transducer $T$ with the same set of states, initial state, and set of final states as $M$. For each transition in $M$ of the form $\delta\left(q_{i},[a, b]\right)=q_{j}$, we define a transition in $T$ from $q_{i}$ to $q_{j}$ with input $a$ and output $b$, except that if $a=\mathrm{B}$, then we set the corresponding transition in $T$ to have input $\epsilon$. Each word that $M$ accepts, having first component $w \mathrm{~B}^{*}$ and second component $y$, corresponds to an input $w$ of $T$ and an output of $y$. The result now follows.

$(\mathrm{e}) \Longrightarrow(\mathrm{c})$ : The construction of the previous paragraph is completely reversible, which shows that $(\mathrm{e}) \Longrightarrow(\mathrm{d})$. But clearly $(\mathrm{d}) \Longrightarrow(\mathrm{c})$.

(c) $\Longrightarrow$ (b): Let $L \subseteq((\Sigma \cup\{\mathrm{B}\}) \times \Delta)^{*}$ be a regular language such that $(f, w)=\left|\left\{z \in L: \tau\left(\pi_{1}(z)\right)=w\right\}\right|$, and let $M=\left(Q, \Sigma^{\prime}, \delta, q_{0}, F\right)$ be a DFA accepting $L$, where $\Sigma^{\prime}=(\Sigma \cup\{\mathrm{B}\}) \times \Delta$. We now create an NFA- $\epsilon M^{\prime}$ with the desired property, by modifying $M$, as follows: first, the set of states is expanded from $Q$ to $Q \times \Delta$. Second, if $M$ has a transition $\delta\left(q_{i},[a, b]\right)=q_{j}$ with $a \in \Sigma$, then $M^{\prime}$ has transitions $\delta\left(\left[q_{i}, c\right], a\right)=\left[q_{j}, b\right]$ for all $c \in \Delta$. Similarly, if $M$ has a transition $\delta\left(q_{i},[\mathrm{~B}, b]\right)=q_{j}$, then $M^{\prime}$ has a transition $\delta\left(\left[q_{i}, c\right], \epsilon\right)=\left[q_{j}, b\right]$ for all $c \in \Delta$. The initial state is $\left[q_{0}, c\right]$ for some arbitrary element $c \in \Delta$, and the set of final states of $M^{\prime}$ is $F \times \Delta$. The formal proof that this works is essentially the proof of (a) $\Longrightarrow$ (b) in Theorem 14 and is omitted. 
(b) $\Longrightarrow$ (a): Given the NFA- $\epsilon M=\left(Q, \Sigma, \delta, q_{0}, F\right)$, we create some associated matrices $D_{a}$ for $a \in \Sigma \cup\{\epsilon\}$. If the set of states $Q=\left\{q_{0}, q_{1}, \ldots, q_{n-1}\right\}$, then $D_{a}$ has a 1 in row $i$ and column $j$ iff $\delta\left(q_{i}, a\right)=q_{j}$.

Now any finite path labeled $w=a_{1} a_{2} \cdots a_{n}$ in the transition diagram of $M$ looks like

$$
\overbrace{\epsilon, \ldots, \epsilon}^{b_{0}}, a_{1}, \overbrace{\epsilon, \ldots, \epsilon}^{b_{1}}, a_{2}, \ldots, \overbrace{\epsilon, \ldots, \epsilon}^{b_{n-1}}, a_{n}, \overbrace{\epsilon, \ldots, \epsilon}^{b_{n}},
$$

for some $b_{0}, b_{1}, \ldots, b_{n}$ with $0 \leq b_{i}<\infty$.

Let $D=\sum_{i \geq 0} D_{\epsilon}^{i}$; this is a matrix with possibly infinite entries. Then the entry in row $i$ and column $j$ of $D D_{a_{1}} D D_{a_{2}} \cdots D D_{a_{n}} D$ gives the number of paths from state $q_{i}$ to state $q_{j}$ in $M$. If $u=\left[\begin{array}{lllll}1 & 0 & 0 & \cdots & 0\end{array}\right]$ and $v$ is the $\{0,1\}$-vector corresponding to the final states of $M$, then $u D D_{a_{1}} D D_{a_{2}} \cdots D D_{a_{n}} D v$ is the number of accepting paths labeled $w$.

If we now define $\mu(a)=D D_{a}$ for $a \in \Sigma$ and $v^{\prime}=D v$, then $\left(u, \mu, v^{\prime}\right)$ is a linear representation for $f$.

\section{Applications to enumeration}

Now that all the basic definitions and results are out of the way, we can resume our work on enumeration. The common theme in what follows is to show that some well-studied sequence is $k$-regular, by combining Theorem 14 or Theorem 21 (which characterize the formal series associated with counting the number of paths, or certain subsets of regular languages, or size of transduced sets, as $\mathbb{N}$ - or $\mathbb{N}_{\infty}$ recognizable) with Theorem 11, which shows the equivalence between $k$-regular sequence and recognizable formal series. Here is a simple example:

Theorem 22. Let $E$ be any finite set of integers, and consider $(b(n))_{n \geq 0}$, the sequence that counts the number of reversed representations of $n$ in base $k$, where the digits are chosen only from $E$, and where reversed representations with trailing zeroes are not allowed. Then $(b(n))_{n \geq 0}$ is $(\mathbb{N}, k)$-regular.

Proof. We construct a transducer $T$ having $b(n)$ distinct outputs on input $(n)_{k}^{R}$. On input $(n)_{k}$, the transducer $T$ guesses a possible representation $w$ using only the digits of $E$, simultaneously "normalizes" it, digit-by-digit, to $w^{\prime}$, and checks that the normalized representation is equal to the input. If it is, then $w$ is output. There are some details to handle if $w$ is shorter or longer than $(n)_{k}^{R}$. If $w$ is shorter, then we allow padding of $w$ with trailing zeroes. If $w$ is longer, then we handle this by permitting $T$ to perform $\epsilon$-transitions on the input after it has processed all the symbols of $(n)_{k}^{R}$.

Then, using Theorem 21 together with Theorem 11 and Corollary 19, we see that $(b(n))_{n \geq 0}$ is an $(\mathbb{N}, k)$-regular sequence.

Example 23. Let $b_{k}(n)$ denote the number of representations of $n$ in base 2, using the digits $\{0,1, \ldots, k-1\}$. Then $b_{2}(n)=1$, from the uniqueness of binary 
representations, and $b_{3}(n)$ is the Stern-Brocot sequence evaluated at $n+1$. From Theorem 22, we see that all these sequences are $\left(\mathbb{N}_{\infty}, 2\right)$-regular. See [29].

We now turn to our main enumeration results.

Theorem 24. Let $\mathbf{x}=a(0) a(1) a(2) \ldots$ be a $k$-automatic sequence. Let $b(n)$ be the number of distinct factors of length $n$ in $\mathbf{x}$. Then $(b(n))_{n \geq 0}$ is an $(\mathbb{N}, k)$-regular sequence.

Proof. To count distinct factors of length $n$, we count the first occurrences of each factor.

The number of distinct factors of length $n$ in $\mathbf{x}$ equals the number of indices $i$ such that there is no index $j<i$ with the factor of length $n$ beginning at position $i$ equal to the factor of length $n$ beginning at position $j$.

Consider the set

$$
\begin{gathered}
S=\{(n, i): \text { for all } j \text { with } 0 \leq j<i \text { there exists an integer } \\
t \quad \text { with } 0 \leq t<n \text { such that } a(i+t) \neq a(j+t)\} .
\end{gathered}
$$

Then, by Theorem 1, the language $S^{\prime}$ defined to be the base- $k$ encoding of elements of $S$, forms a regular language. We assume without loss of generality that if one representation of $(n, i)$ appears in $S^{\prime}$, then they all do, including the ones with leading (actually, trailing zeroes).

We now apply a transducer to $S^{\prime}$, changing every representation of $(n, i)$ as follows: we change every 0 after the last nonzero digit in the first component to B. This transformation preserves the regularity of $S^{\prime}$. Finally, we discard every representation that ends with $[\mathrm{B}, 0]$. The effect of this is to ensure that $n$ in the first component, up to ignoring the B's, has a single representation, and that each $i$ corresponding to a particular $n$ has a unique representation. Using Theorems 14 and 11, we see that $(b(n))_{n \geq 0}$ is $(\mathbb{N}, k)$-regular.

Remark 25. Mossé 27] proved, among other things, that a sequence that is the fixed point of a $k$-uniform morphism has a $k$-regular subword complexity function. With our technique, we obtain her result for these sequences and also the slightly more general case of $k$-automatic sequence.

Theorem 26. The sequence counting the number of palindromic factors of length $n$ is $(\mathbb{N}, k)$-regular.

Proof. The number of distinct palindromes of length $n$ in $\mathbf{x}$

is equal to

the number of indices $i$ such that $\mathbf{x}[i . . i+n-1]$ is a palindrome and $\mathbf{x}[i . . i+n-1]$ does not appear previously in $\mathbf{x}$

is equal to 
the number of indices $i$ such that $\mathbf{x}[i . . i+n-1]=\mathbf{x}[i . . i+n-1]^{R}$ and for all $j$ with $0 \leq j<i, \mathbf{x}[i . . i+n-1]$ is not the same as $\mathbf{x}[j . . j+n-1]$

is equal to the number of indices $i$ such that for all $t, 0 \leq t \leq n / 2, a(i+t)=a(i+n-1-t)$ and for all $j$ with $0 \leq j<i$, there exists $u$ with $0 \leq u<n$ such that $a(i+u) \neq a(j+u)$. Now apply Theorems 14 and 11 .

Remark 27. Allouche, Baake, Cassaigne, and Damanik [1, Thm. 10, proved that the palindrome complexity of the fixed point of a primitive $k$-uniform morphism is $k$-automatic. Our result is more general: it shows that the palindrome complexity of a $k$-automatic sequence is $k$-regular, and hence is $k$-automatic iff it is bounded.

Jean-Paul Allouche kindly informs us that our result has just been obtained independently by Carpi and D'Alonzo [12].

Example 28. Let $f(n)$ denote the number of unbordered factors of length $n$ of the Thue-Morse sequence. Here is a brief table of the values of $f(n)$ :

\begin{tabular}{|c|c|c|c|c|c|c|c|c|c|c|c|c|c|c|c|c|}
\hline$n$ & 1 & 2 & 3 & 4 & 5 & 6 & 7 & 8 & 9 & 10 & 11 & 12 & 13 & 14 & 15 & 16 \\
\hline$f(n)$ & 2 & 2 & 4 & 2 & 4 & 6 & 0 & 4 & 4 & 4 & 4 & 12 & 0 & 4 & 4 & 8 \\
\hline
\end{tabular}

By Theorems 14 and 11 we know that $f$ is ( $\mathbb{N}, 2)$-regular. Conjecturally, $f$ is given by the system of recurrences

$$
\begin{aligned}
f(4 n+1) & =f(2 n+1) \\
f(8 n+2) & =f(2 n+1)-8 f(4 n)+f(4 n+3)+4 f(8 n) \\
f(8 n+3) & =2 f(2 n)-f(2 n+1)+5 f(4 n)+f(4 n+2)-3 f(8 n) \\
f(8 n+4) & =-4 f(4 n)+2 f(4 n+2)+2 f(8 n) \\
f(8 n+6) & =2 f(2 n)-f(2 n+1)+f(4 n)+f(4 n+2)+f(4 n+3)-f(8 n) \\
f(16 n) & =-2 f(4 n)+3 f(8 n) \\
f(16 n+7) & =-2 f(2 n)+f(2 n+1)-5 f(4 n)+f(4 n+2)+3 f(8 n) \\
f(16 n+8) & =-8 f(4 n)+4 f(4 n+2)+4 f(8 n) \\
f(16 n+15) & =-8 f(4 n)+2 f(4 n+3)+4 f(8 n)+f(8 n+7) .
\end{aligned}
$$

In principle this could be verified by our method, but we have not yet done so.

Theorem 29. Let $\mathbf{x}=a(0) a(1) a(2) \cdots$ be a $k$-automatic sequence. Then the following sequences are also $k$-automatic:

(a) $b(i)=1$ if there is a square beginning at position $i$; 0 otherwise

(b) $c(i)=1$ if there is a square centered at position $i$; 0 otherwise

(c) $d(i)=1$ if there is an overlap beginning at position $i$; 0 otherwise 
(d) $e(i)=1$ if there is a palindrome beginning at position $i$; 0 otherwise

(e) $f(i)=1$ if there is a palindrome centered at position $i$; 0 otherwise

Remark 30. Brown, Rampersad, Shallit, and Vasiga proved results (a)-(c) for the special case of the Thue-Morse sequence 8$]$.

Theorem 31. Let $\mathbf{x}$ and $\mathbf{y}$ be $k$-automatic sequences. Then the following are $\left(\mathbb{N}_{\infty}, k\right)$-regular:

(a) the number of distinct square factors in $\mathbf{x}$ of length $n$;

(b) the number of squares in $\mathbf{x}$ beginning at (centered at, ending at) position $n$;

(c) the length of the longest square in $\mathbf{x}$ beginning at (centered at, ending at) position $n$;

(d) the number of palindromes in $\mathbf{x}$ beginning at (centered at, ending at) position $n$;

(e) the length of the longest palindrome in $\mathbf{x}$ beginning at (centered at, ending at) position n;

(f) the length of the longest fractional power in $\mathbf{x}$ beginning at (ending at) position $n$;

(g) the number of distinct recurrent factors in $\mathbf{x}$ of length $n$;

(h) the number of factors of length $n$ that occur in $\mathbf{x}$ but not in $\mathbf{y}$.

(i) the number of factors of length $n$ that occur in both $\mathbf{x}$ and $\mathbf{y}$.

Remark 32. Brown, Rampersad, Shallit, and Vasiga proved results (b)-(c) for the special case of the Thue-Morse sequence [ $\underline{8}$.

We now turn to some other measures that have received much attention. The recurrence function $R_{\mathbf{x}}(n)=R(n)$ of an infinite word $\mathbf{x}$ is the smallest integer $t$ such that every factor of length $t$ of $\mathbf{x}$ contains as a factor every factor of length $n$. Said otherwise, it is the size of the smallest "window" one can slide along $\mathbf{x}$ and always contain all length- $n$ factors.

Theorem 33. If $\mathbf{x}$ is k-automatic, then $\left(R_{\mathbf{x}}(n)\right)_{n \geq 0}$ is $\left(\mathbb{N}_{\infty}, k\right)$-regular.

Proof. We translate the predicate " $R(n)>t$ ", as follows:

$R(n)>t$

iff

there exists $i \geq 0, j \geq 0$ such that $\mathbf{x}[j . . j+n-1]$ appears nowhere in $\mathbf{x}[i . . i+t-1]$

iff

there exists $i \geq 0, j \geq 0$ such that for all integers $l$ with $i \leq l<i+t-1-n$ we have $\mathbf{x}[l . . l+n-1] \neq \mathbf{x}[j . . j+n-1]$

iff

there exists $i \geq 0, j \geq 0$, such that for all integers $l$ with $i \leq l<i+t-1-n$ there exists $m, 0 \leq m<n$ such that $\mathbf{x}[l+m] \neq \mathbf{x}[j+m]$. 
Now for any fixed $n$, the number of positive integers $t$ for which $R(n)>t$ is equal to $R(n)$. Hence $(R(n))_{n \geq 0}$ is $\left(\mathbb{N}_{\infty}, k\right)$-regular.

Another measure is called "appearance" [5], §10.10. The appearance function $A_{\mathbf{x}}(n)=A(n)$ is the smallest integer $t$ such that every factor of length $n$ appears in a prefix of length $t$ of $\mathbf{x}$. The following result can be proved in an analogous manner to the previous one.

Theorem 34. If $\mathbf{x}$ is $k$-automatic, then $\left(A_{\mathbf{x}}(n)\right)_{n \geq 0}$ is $(\mathbb{N}, k)$-regular.

Next, we consider a measure due to Garel [21]. The separator length $S_{\mathbf{x}}(n)$ is the length of the smallest factor that begins at position $n$ of $\mathbf{x}$ and does not occur previously.

Theorem 35. If $\mathbf{x}$ is k-automatic, then $\left(S_{\mathbf{x}}(n)\right)_{n \geq 0}$ is $(\mathbb{N}, k)$-regular.

Proof. The predicate " $S_{\mathbf{x}}(n)>t$ " is the same as saying that for every $i \leq t$ the word of length $i$ beginning at position $n$ of $\mathbf{x}$ occurs previously in $\mathbf{x}$, which is the same as saying for all $i, 0 \leq i \leq t$, there exists $j, 0 \leq j<n$ such that $\mathbf{x}[n . . n+i-1]=\mathbf{x}[j . . j+i-1]$. Now look at the pairs $(n, t)$ satisfying this, with $n$ positive. For each $n$ there are exactly $S_{\mathbf{x}}(n)$ different $t$ 's that work.

Remark 36. Garel 21] proved this for the case of a fixed point of a uniform circular morphism; our proof works for the more general case of an arbitrary $k$-automatic sequence.

Carpi and D'Alonzo have introduced a measure they called repetitivity index [11. This measure $I_{\mathbf{x}}(n)$ is the minimum distance between two consecutive occurrences of the same length- $n$ factor in $\mathbf{x}$. But " $I_{\mathbf{x}}(n)>t$ " is the same as saying for all $i, j \geq 0$ with $i \neq j$, the equality $\mathbf{x}[i . . i+n-1]=\mathbf{x}[j . . j+n-1]$ implies that $j-i>t$. Hence we get

Theorem 37. If $\mathbf{x}$ is $k$-automatic, then its repetitivity index is $(\mathbb{N}, k)$-regular.

For our final application, Frid and Zamboni [19] introduced the notion of "automatic permutation". This is a permutation of $\mathbb{N}$ based on a $k$-automatic sequence $\mathbf{x}$, as follows: we say $i<j$ if the infinite word $\mathbf{x}[i . . \infty]$ is lexicographically less than the word $\mathbf{x}[j . . \infty]$. The permutation complexity $p_{\mathbf{x}}(n)$ is the map that sends $n$ to the number of distinct finite permutations of length $n$ induced by $\mathbf{x}[18$.

Theorem 38. The permutation complexity of a $k$-automatic sequence is $(\mathbb{N}, k)$ regular.

Proof. First, we need to see that for $k$-automatic sequences the predicate "the shift of $\mathbf{x}$ beginning at position $i$ is lexicographically less than the shift beginning at position $j "$ is $k$-automatic. 
To see this, given positions $i$ and $j$, we verify that there is some index $t$ such that $a[i+l]=a[j+l]$ for all $l<t$, and also that $a[i+t]<a[j+t]$.

Next, we need to see that given $i, j, n$ we need to see that the predicate "the length- $n$ permutation induced by the shifts starting at position $i$ coincides with that starting at $j "$ is automatic.

To do this we verify that for all indices $l$ with $i \leq l, m<i+n$, the relation in the previous paragraph holds between $i+l$ and $i+m$ in the same way as it holds for $j+l$ and $j+m$.

In the final step, we enumerate the number of indices $i$ for which the permutation at position $i$ of length $n$ does not match the one occurring at any previous index. This is just the number of distinct permutations of length $n$.

As a corollary, we recover the result of Widmer 35] that the permutation complexity of the Thue-Morse word is (N, 2)-regular. In principle his description could be mechanically verified.

\section{Linear bounds}

Yet another application of our method allows us to obtain linear bounds on many quantities associated with automatic sequences. As a first example, we recover an old result of Cobham [14] on "subword" complexity.

Theorem 39. The number of distinct factors of length $n$ of an automatic sequence is $O(n)$.

Proof. Let $\mathbf{x}$ be a $k$-automatic sequence. By Theorem 1 we know that the base- $k$ encoding $S^{\prime}$ of

$$
\begin{gathered}
S=\{(n, I): \text { for all } j<I \text { the factor of length } n \text { starting at position } j \\
\text { is different from the one starting at position } I\}
\end{gathered}
$$

is a regular language.

Suppose that the factor complexity of $\mathbf{x}$ is not $O(n)$. Then for every $L$ there exists some pair $(n, I) \in S$ such that the length of the canonical encoding of $I$ is longer than that of $n$ by at least $L$ digits. So in $S^{\prime}$ there is some word of the form $(n)_{k} \mathrm{~B} \geq L \times(I)_{k}$, where $(u)_{k}$ denotes the canonical encoding of $u$ in base $k$ and $\times$ is how we join separate components to form a word.

Since the length of $(I)_{k}$ is very much longer than that of $(n)_{k}$, we can apply the pumping lemma to this word, where we only pump in the portion of $(I)_{k}$ that is longer than $(n)_{k}$. Hence when we pump, we only add B's to the first component, and so its value remains unchanged. In this way by pumping we obtain infinitely many values $I^{\prime}$ such that $\left(n, I^{\prime}\right) \in S$. In other words, there are infinitely many distinct factors of length $n$, which is clearly absurd. The contradiction proves the result.

In a similar manner we can prove that all the quantities in Theorem 31 are either linearly bounded, or unbounded. 


\section{Other numeration systems}

All our results transfer, mutatis mutandis, to the setting of other numeration systems where addition can be performed on numbers using a transducer that processes numbers starting with the least significant digit.

A (generalized) numeration system is given by an increasing sequence of integers $U=\left(U_{i}\right)_{i \geq 0}$ such that $U_{0}=1$ and $C_{U}:=\lim _{i \rightarrow+\infty} U_{i+1} / U_{i}$ exists and is finite. Then the canonical $U$-representation of $n$ (with least significant digit first), which is denoted by $(n)_{U}$, is the unique finite word $w$ over the alphabet $\Sigma_{U}=\left\{0, \ldots, C_{U}-\right.$ $1\}$ not ending with 0 and satisfying $n=\sum_{i=0}^{|w|-1} w[i] U_{i}$ and $\forall t \in\{0, \ldots,|w|-$ $1\}, \sum_{i=0}^{t} w[i] U_{i}<U_{t+1}$. The notion of $k$-automatic sequence extends naturally to this context: an infinite sequence $\mathbf{x}$ is said to be $U$-automatic if it is computable by a finite automaton taking as input the $U$-representation $(n)_{U}$ of $n$, and having $\mathbf{x}[n]$ as the output associated with the last state encountered.

A numeration system $U$ is called linear if $U$ satisfies a linear recurrence relation over $\mathbb{Z}$. A Pisot system is a linear numeration system $U$ whose characteristic polynomial is the minimal polynomial of a Pisot number. Recall that a Pisot number is an algebraic integer greater than 1, all of whose conjugates have moduli less than 1. For example, all integer base numeration systems and the Fibonacci numeration system are Pisot systems. Frougny and Solomyak [20] proved that addition is $U$-recognizable within all Pisot systems $U$, i.e., it can be performed by a finite letter-to-letter transducer reading $U$-representations with least significant digit first. Bruyère and Hansel [9] then proved the following logical characterization of $U$-automatic sequences for Pisot systems: a sequence is $U$-automatic if and only if it is $U$-definable, i.e., it is expressible as a predicate of $\left\langle\mathbb{N},+, V_{U}\right\rangle$, where $V_{U}(n)$ is the smallest $U_{i}$ occurring in $(n)_{U}$ with a nonzero coefficient. Therefore, if $U$ is a Pisot system, any combinatorial property of $U$-automatic words that can be described by a predicate of $\left\langle\mathbb{N},+, V_{U}\right\rangle$ is decidable.

The notion of $(R, k)$-regular sequences extends to Pisot numeration systems: an infinite sequence $\mathbf{x}$ is said to be $(R, U)$-regular if the series $\sum_{n \geq 0} \mathbf{x}[n](n)_{U}$ is an $R$-recognizable series. Thus we obtain

Theorem 40. Let $U$ be a Pisot numeration system and let $\mathbf{x}$ be any $U$-automatic word. The following sequences are $U$-automatic:

(a) $a(n)=1$ if there is a square beginning at (centered at, ending at) position $n$ of $\mathbf{x}, 0$ otherwise;

(b) $b(n)=1$ if there is a palindrome beginning at (centered at, ending at) position $n$ of $\mathbf{x}, 0$ otherwise;

(c) $c(n)=1$ if there is an unbordered factor beginning at (centered at, ending at) position $n$ of $\mathbf{x}, 0$ otherwise.

The following sequences are $\left(\mathbb{N}_{\infty}, U\right)$-regular:

(a) The number of distinct square factors beginning at (centered at, ending at) 
position $n$ of $\mathbf{x}$;

(b) The number of distinct palindromic factors beginning at (centered at, ending at) position $n$ of $\mathbf{x}, 0$ otherwise;

(c) The number of distinct unbordered factors beginning at (centered at, ending at) position $n$ of $\mathbf{x}, 0$ otherwise.

Berstel showed that the cardinality of the set of unnormalized Fibonacci representations is Fibonacci-regular [6], a result also obtained (but not published) by the third author about the same time. In analogy with Theorem 22 we have

Theorem 41. The number of unnormalized representations of $n$ in a Pisot numeration system $U$ is $\left(\mathbb{N}_{\infty}, U\right)$-regular.

\section{Closing remarks}

It may be worth noting that the explicit constructions of automata we have given also imply bounds on the smallest example of (or counterexample to) the properties we consider. The bounds are essentially given by a tower of exponents whose height is related to the number of alternating quantifiers. For example,

Theorem 42. Suppose $\mathbf{x}$ and $\mathbf{y}$ are $k$-automatic sequences generated by automata with at most $q$ states. If the set of factors of $\mathbf{x}$ differs from the set of factors of $\mathbf{y}$, then there exists a factor of length at most $2^{2^{2^{2 q^{2}}}}$ that occurs in one word but not the other.

We also note that a question left open in [2, regarding the description of the lexicographically least word in the orbit closure of the Rudin-Shapiro sequence, was recently solved by Currie [15].

Finally, in a recent paper [34, the third author shows that additional properties of automatic sequences are deducible by expanding on the techniques in this paper. For example, the critical exponent is computable.

\section{Acknowledgments}

We thank Jean-Paul Allouche for his helpful comments.

\section{References}

[1] J.-P. Allouche, M. Baake, J. Cassaigne, and D. Damanik. Palindrome complexity. Theoret. Comput. Sci. 292 (2003), 9-31.

[2] J.-P. Allouche, N. Rampersad, and J. Shallit. Periodicity, repetitions, and orbits of an automatic sequence. Theoret. Comput. Sci. 410 (2009), 2795-2803.

[3] J.-P. Allouche and J. O. Shallit. The ring of $k$-regular sequences. Theoret. Comput. Sci. 98 (1992), 163-197.

[4] J.-P. Allouche and J. O. Shallit. The ring of $k$-regular sequences, II. Theoret. Comput. Sci. 307 (2003), 3-29. 
[5] J.-P. Allouche and J. Shallit. Automatic Sequences: Theory, Applications, Generalizations. Cambridge University Press, 2003.

[6] J. Berstel. An exercise on Fibonacci representations. RAIRO Inform. Théor. App. 35 (2001), 491-498.

[7] J. Berstel and C. Reutenauer. Noncommutative Rational Series With Applications, Vol. 137 of Encyclopedia of Mathematics and Its Applications. Cambridge University Press, 2011.

[8] S. Brown, N. Rampersad, J. Shallit, and T. Vasiga. Squares and overlaps in the Thue-Morse sequence and some variants. RAIRO Inform. Théor. App. 40 (2006), 473-484.

[9] V. Bruyère and G. Hansel. Bertrand numeration systems and recognizability. Theoret. Comput. Sci. 181 (1997), 17-43.

[10] V. Bruyère, G. Hansel, C. Michaux, and R. Villemaire. Logic and p-recognizable sets of integers. Bull. Belgian Math. Soc. 1 (1994), 191-238. Corrigendum, Bull. Belg. Math. Soc. 1 (1994), 577.

[11] A. Carpi and V. D'Alonzo. On the repetitivity index of infinite words. Internat. J. Algebra Comput. 19 (2009), 145-158.

[12] A. Carpi and V. D'Alonzo. On factors of synchronized sequences. To appear, Theor. Comput. Sci., 2011.

[13] A. Carpi and C. Maggi. On synchronized sequences and their separators. RAIRO Inform. Théor. App. 35 (2001), 513-524.

[14] A. Cobham. Uniform tag sequences. Math. Systems Theory 6 (1972), 164-192.

[15] J. D. Currie. Lexicographically least words in the orbit closure of the Rudin-Shapiro word. http://arxiv.org/pdf/0905.4923, 2010.

[16] J. D. Currie and K. Saari. Least periods of factors of infinite words. RAIRO Inform. Théor. App. 43 (2009), 165-178.

[17] I. Fagnot. Sur les facteurs des mots automatiques. Theoret. Comput. Sci. 172 (1997), $67-89$.

[18] A. Frid. Infinite permutations vs. infinite words. In P. Ambrož, S. Holub, and Z. Masáková, editors, WORDS 2011, 8th International Conference. Elect. Proc. Theor. Comput. Sci., 2011. Available at http://arxiv.org/abs/1108.3616v1

[19] A. Frid and L. Q. Zamboni. On automatic infinite permutations. Presented at Journées Montoises, 2010.

[20] C. Frougny and B. Solomyak. On representation of integers in linear numeration systems. In M. Pollicott and K. Schmidt, editors, Ergodic Theory of $\mathbb{Z}^{d}$ Actions (Warwick, 1993-1994), Vol. 228 of London Mathematical Society Lecture Note Series, pp. 345-368. Cambridge University Press, 1996.

[21] E. Garel. Séparateurs dans les mots infinis engendrés par morphismes. Theoret. Comput. Sci. 180 (1997), 81-113.

[22] V. Halava, T. Harju, T. Kärki, and M. Rigo. On the periodicity of morphic words. In Developments in Language Theory 2010, Vol. 6224 of Lecture Notes in Computer Science, pp. 209-217. Springer-Verlag, 2010.

[23] J. Honkala. A decision method for the recognizability of sets defined by number systems. RAIRO Inform. Théor. App. 20 (1986), 395-403.

[24] D. Krieger and J. Shallit. Every real number greater than 1 is a critical exponent. Theoret. Comput. Sci. 381 (2007), 177-182.

[25] W. Kuich and A. Salomaa. Semirings, Automata, Languages. Springer-Verlag, 1986.

[26] J. Leroux. A polynomial time Presburger criterion and synthesis for number decision diagrams. In 20th IEEE Symposium on Logic in Computer Science (LICS 2005), pp. 147-156. IEEE Press, 2005. 
[27] B. Mossé. Reconnaissabilité des substitutions et complexité des suites automatiques. Bull. Soc. Math. France 124 (1996), 329-346.

[28] F. Nicolas and Yu. Pritykin. On uniformly recurrent morphic sequences. Internat. J. Found. Comp. Sci. 20 (2009), 919-940.

[29] B. Reznick. Some binary partition functions. In Analytic Number Theory, Vol. 85 of Progr. Math., pp. 451-477. Birkhäuser, 1990.

[30] K. Saari. On the Frequency and Periodicity of Infinite Words. PhD thesis, University of Turku, Finland, 2008.

[31] J. Sakarovitch. Elements of Automata Theory. Cambridge University Press, 2009.

[32] A. Salomaa and M. Soittola. Automata-Theoretic Aspects of Formal Power Series. Springer-Verlag, 1978.

[33] M.-P. Schützenberger. On a theorem of R. Jungen. Proc. Amer. Math. Soc. 13 (1962), 885-890.

[34] J. Shallit. The critical exponent is computable for automatic sequences. In P. Ambrož, S. Holub, and Z. Masáková, editors, WORDS 2011, 8th International Conference. Elect. Proc. Theor. Comput. Sci., 2011. Available at http://arxiv.org/abs/1104.2303v2

[35] S. Widmer. Permutation complexity of the Thue-Morse word. Adv. in Appl. Math. 47 (2011), 309-329. 